\title{
The two-step average tree value for graph and hypergraph games*
}

\author{
Liying Kang ${ }^{\dagger} \quad$ Anna Khmelnitskaya $^{\ddagger} \quad$ Erfang Shan $^{\S}$ \\ Dolf Talman $\quad$ Guang Zhang $\|$
}

June 24, 2020

\begin{abstract}
We introduce the two-step average tree value for transferable utility games with restricted cooperation represented by undirected communication graphs or hypergraphs. The solution can be considered as an alternative for both the average tree solution for graph games and the average tree value for hypergraph games. Instead of averaging players' marginal contributions corresponding to all admissible rooted spanning trees of the underlying (hyper)graph, which determines the average tree solution or value, we consider a two-step averaging procedure, in which in the first step for each player the average of players' marginal contributions corresponding to all admissible rooted spanning trees that have this player as the root is calculated, and in the second step the average over all players of all the payoffs obtained in the first step is computed. In general these two approaches lead to different solution concepts. When each component in the underlying communication structure is cycle-free, a linear cactus with cycles, or the complete graph, the two-step average tree value coincides with the average tree value. A comparative analysis of both solution concepts is done and an axiomatization of the the two-step average tree value on the subclass of TU games with semi-cycle-free hypergraph communication structure, which is more general than that given by a cycle-free hypergraph, is obtained.
\end{abstract}

Keywords: TU game; hypergraph communication structure; average tree value; component fairness

${ }^{*}$ The research of Anna Khmelnitskaya was supported by RFBR (Russian Foundation for Basic Research) grant \#18-01-00780. Her research was done partially during her stay at the University of Twente, whose hospitality is highly appreciated. The research of Guang Zhang was supported by NSFC (National Natural Science Foundation of China) grant \#71901145.

${ }^{\dagger}$ L. Kang, Department of Mathematics, Shanghai University, 200444, Shanghai, P.R.China, e-mail: lykang@i.shu.edu.cn

${ }^{\ddagger}$ A.B. Khmelnitskaya, Saint Petersburg State University, 7/9 Universitetskaya nab., Saint Petersburg 199034, Russia \& V.A. Trapeznikov Institute of Control Sciences of the Russian Academy of Sciences, 65 Profsoyuznaya st., Moscow 117997, Russia, e-mail: a.b.khmelnitskaya@utwente.nl

${ }^{\S}$ E. Shan, School of Management, Shanghai University, 200444, Shanghai, P.R.China, e-mail: efshan@shu.edu.cn

^A.J.J. Talman, CentER, Department of Econometrics and Operations Research, Tilburg University, P.O. Box 90153, 5000 LE Tilburg, The Netherlands, e-mail: talman@tilburguniversity.edu

"G. Zhang, Business School, University of Shanghai for Science and Technology, 200093, Shanghai, P.R.China, e-mail: g.zhang@usst.edu.cn 


\section{JEL Classification Number: C71 \\ Mathematics Subject Classification 2000: 91A12, 91A43}

\section{Introduction}

In classical cooperative game theory it is assumed that any coalition of players may form and realize its worth, and fair distribution of total rewards among the players takes into account capacities of all coalitions. For example, the most prominent solution of cooperative games with transferable utility, or TU games, the Shapley value, cf. Shapley (1953), assigns to each player as a payoff the average of the player's marginal contributions to his predecessors with respect to all possible linear orderings of the players. However, in many practical situations the collection of feasible coalitions is restricted by some social, economical, communication, or technical structure. The study of transferable utility games with limited cooperation introduced by means of an undirected communication graph, called for brevity graph games, is initiated in Myerson (1977). Assuming that only connected players can cooperate, the Myerson value for graph games is defined as the Shapley value of the so-called restricted game for which the worth of each coalition is equal to the sum of the worths of its connected components in the graph. Lately several other solutions for graph games based also on Myerson's assumption that only connected players can cooperate are proposed, in particular, the average tree solution, introduced by Herings, van der Laan, and Talman, cf. Herings et al. (2008), for cycle-free graph games and generalized by Herings, van der Laan, Talman, and Yang, cf. Herings et al. (2010), for the class of all graph games. In comparison to the Myerson value the average tree solution is stable on the subclass of superadditive cycle-free graph games and for cycle-free graph games the order of computational complexity of the average tree solution is linear in the number of players, while it is exponential for the Myerson value.

Yet, the communication graphs reflect only bilateral communication between the players. The idea of consideration of cooperative games with a more general communication structure, allowing to represent communication within sets of more than two players appears first in Myerson (1980), where NTU games with conference structure are investigated. In fact a conference in terms of Myerson coincides with a hyperlink of a hypergraph. TU games with hypergraph communication structure, called for brevity hypergraph games, are formally introduced by van den Nouweland, Borm, and Tijs, cf. van den Nouweland et al. (1992), where also the Myerson and position values ${ }^{1}$ for hypergraph games are defined and axiomatized. Recently the average tree value for hypergraph games, which generalizes the average tree solution for graph games to hypergraph games, has been introduced and investigated by Kang, Khmelnitskaya, Shan, Talman, and Zhang in Kang et al. (2020).

The goal of this paper is to introduce a two-step average tree value for hypergraph games, and in particular for graph games, which can be considered as an alternative to the average tree value. Similar to the average tree value for graph and hypergraph games, the new solution is based on the idea that the payoff to a player is determined by averaging of the player's marginal contributions with respect to all

\footnotetext{
${ }^{1}$ The position value for graph games is first defined in Meessen (1988) and later studied and axiomatized by Borm, Owen, and Tijs, cf. Borm et al. (1992).
} 
admissible rooted spanning trees of the given communication structure. Since in general for distinct players the numbers of admissible rooted spanning trees having these players as the roots might be different, the simultaneous averaging via all admissible rooted spanning trees unwittingly implies that the players, being the roots of admissible rooted spanning trees, participate in the definition of the average tree value with weights determined by the numbers of admissible rooted spanning trees having the corresponding players as their roots. The latter in a sense conflicts with the idea that every player in a (hyper)graph game is equally important. To eliminate this drawback we consider a two-step averaging procedure, in which in the first step for each player the average of players' marginal contributions corresponding to all admissible rooted spanning trees having this player as the root is calculated, and in the second step the average over all players of all the payoffs obtained in the first step is computed. A comparative analysis of both average tree solution concepts shows that the better structured averaging procedure underlying the two-step average tree value provides it with additional attractive properties that are valid also on wider subclasses of hypergraph games. Furthermore, an axiomatization of the two-step average tree value on the class of semi-cycle-free hypergraph games, which includes all cycle-free hypergraph games as a proper subclass, is provided, and its core stability for superadditive quasi-cycle-free hypergraph games is obtained.

The paper is organized as follows. Basic definitions and notation are given in Section 2. In Section 3 the two-step average tree value for (hyper)graph games is introduced and the cases of its coincidence with the average tree value are investigated. Section 4 is devoted to study the properties of the two-step average tree value in comparison with the properties of the average tree value. Section 5 provides an axiomatic characterization of the two-step average tree value. Section 6 examines the core stability.

\section{Preliminaries}

A cooperative game with transferable utility, or $T U$ game, is a pair $(N, v)$, where $N=\{1,2, \ldots, n\}$ is a finite set of $n$ players and $v: 2^{N} \rightarrow \mathbb{R}$ is a characteristic function, with $v(\emptyset)=0$, assigning to every coalition $S \subseteq N$ its worth $v(S)$, which can be freely distributed as payoff among the members of $S$. We denote by $\mathcal{G}_{N}$ the set of TU games with fixed player set $N$. For simplicity of notation and if no ambiguity appears we write $v$ instead of $(N, v)$. A game $v \in \mathcal{G}_{N}$ is superadditive if $v(S \cup Q) \geq v(S)+v(Q)$ for all $S, Q \subseteq N$ satisfying $S \cap Q=\emptyset$. The unanimity game with respect to coalition $S \in 2^{N} \backslash\{\emptyset\}$ is the game $u_{S} \in \mathcal{G}_{N}$ defined as $u_{S}(Q)=1$ if $S \subseteq Q$ and 0 otherwise. For a finite set $S,|S|$ denotes the cardinality of $S$.

A communication structure on the set of players $N$ is specified by a graph or hypergraph on $N$. A hypergraph on $N$ is a set $H \subseteq\left\{e \in 2^{N}|| e \mid \geq 2\right\}$ of hyperlinks. A hypergraph $H$ is $r$-uniform if $|e|=r$ for all $e \in H$. A 2-uniform hypergraph on $N$ is an (undirected) graph on $N$ and is denoted by a set of links $\Gamma \subseteq \Gamma^{N}$, where $\Gamma^{N}=\{\{i, j\} \mid i, j \in N, i \neq j\}$ is the complete graph on $N$. We denote by $\mathcal{H}_{N}\left(\Gamma_{N}\right)$ the set of hypergraphs (graphs) on $N$.

Let $H \in \mathcal{H}_{N}$. For $i \in N, H_{i}=\{e \in H \mid e \ni i\}$ is the set of hyperlinks in $H$ containing $i$ with $\left|H_{i}\right|$ the degree of $i$ in $H$. A player $i$ is non-connective in $H$ if $\left|H_{i}\right| \leq 1$, otherwise player $i$ is connective in $H$. Two players $i$ and $j$ are interactive in 
$H$ if $H_{i} \neq \emptyset$ and $H_{j}=H_{i}$. A player $j$ is adjacent to player $i$ in $H$ if $i, j \in e$ for some $e \in H$. A sequence $C=\left(i_{1}, e_{1}, i_{2}, e_{2}, \ldots, i_{k-1}, e_{k-1}, i_{k}\right)$, with $k \geq 2$, is a chain in $H$ between player $i_{1}$ and player $i_{k}$ if it satisfies the following conditions: (i) $i_{1}, \ldots, i_{k-1}$ are distinct players in $N$, (ii) $i_{2}, \ldots, i_{k}$ are distinct players in $N$, (iii) $e_{1}, \ldots, e_{k-1}$ are distinct hyperlinks in $H$, and (iv) $i_{t+1}, i_{t} \in e_{t}$ for all $t \in\{1, \ldots, k-1\}$. For a chain $C=\left(i_{1}, e_{1}, i_{2}, e_{2}, \ldots, i_{k-1}, e_{k-1}, i_{k}\right)$ in $H, N(C)=\bigcup_{t=1}^{k-1} e_{t}$ is the set of players contained in $C$ and the sequence $\left(i_{1}, i_{2}, \ldots, i_{k}\right)$ is a path in $H$ between players $i_{1}$ and $i_{k} . H$ is connected if $n=1$ or there exists a chain in $H$ between every two distinct players in $N$. A chain $\left(i_{1}, e_{1}, i_{2}, e_{2}, \ldots, i_{k-1}, e_{k-1}, i_{k}\right)$ in $H$ is a cycle in $H$ if $k \geq 3$ and $i_{1}=i_{k} . H$ is cycle-free if there is no cycle in $H$ and $H$ is linear if $\left|e \cap e^{\prime}\right| \leq 1$ for every two distinct $e, e^{\prime} \in H$. Note that a cycle-free hypergraph is linear, because $\left\{i_{1}, i_{2}\right\} \subseteq e_{1} \cap e_{2}, e_{1} \neq e_{2}$, implies that $\left(i_{1}, e_{1}, i_{2}, e_{2}, i_{1}\right)$ is a cycle. $H$ is a cactus if $H$ is connected and any two distinct cycles in $H$ have at most one player in common, i.e., $\left|N(C) \cap N\left(C^{\prime}\right)\right| \leq 1$ for every two distinct cycles $C, C^{\prime}$ in $H$. Note that a connected cycle-free hypergraph is a linear cactus, but a cactus might be nonlinear as well, for example, $H=\left\{e_{1}, e_{2}\right\}$, where $e_{1}=\{1,2,3\}$ and $e_{2}=\{2,3,4\}$.

For $S \subseteq N,\left.H\right|_{S}=\{e \in H \mid e \subseteq S\}$ is the subhypergraph of $H$ induced by $S$. A coalition $S \subseteq N$ is connected in $H$ if $\left.H\right|_{S}$ is connected, i.e., $|S|=1$ or there exists a chain in $\left.H\right|_{S}$ between every two distinct players in $S . C^{H}(S)$ denotes the set of subsets of $S \subseteq N$ that are connected in $H$. For $S \subseteq N, Q$ is a component of $S$ in $H$, if $Q$ is a maximal connected subset of $S$ in $H$. $S / H$ denotes the set of components of $S \subseteq N$ in $H$. A hyperlink $e \in H$ is a bridge in $H$ if $|N / H|<|N /(H \backslash\{e\})|$.

A rooted tree on a component $K \in N / H$ of $N$ in $H$ is a set $T \subseteq\{(i, j) \mid i, j \in K, i \neq$ $j$ \} of directed links with one player $r(T)$, the root of $T$, satisfying that $(i, r(T)) \notin T$ for all $i \in K$ and for every $i \in K, i \neq r(T)$, there is a unique directed path $\left(i_{1}, \ldots, i_{k}\right)$ in $T$ from $i_{1}$ to $i_{k}$, where $i_{1}=r(T), i_{k}=i$, and $\left(i_{h}, i_{h+1}\right) \in T$ for all $h \in\{1, \ldots, k-1\}$. If there exists a directed path in $T$ from $i$ to $j$, then $j$ is a successor of $i$ and $i$ is a predecessor of $j$ in $T$, and if $(i, j) \in T$, then $j$ is an immediate successor of $i$ and $i$ is an immediate predecessor of $j$ in $T$. For $i \in K, S_{i}^{T}$ and $\widehat{S}_{i}^{T}$ denote the set of successors and the set of immediate successors of $i$ in $T$, respectively, and $\bar{S}_{i}^{T}=S_{i}^{T} \cup\{i\}$. T is a rooted spanning tree of $\left.H\right|_{K}$ if $(i, j) \in T$ implies $\{i, j\} \subseteq e$ for some $\left.e \in H\right|_{\bar{S}_{i}^{T}}$. A rooted spanning tree $T$ of $\left.H\right|_{K}$ is admissible if $(i, j) \in T$ implies $\bar{S}_{j}^{T} \in S_{i}^{T} / H . \mathcal{T}^{H}(K)$ denotes the set of admissible rooted spanning trees of $\left.H\right|_{K}$ and, for $r \in K, \mathcal{T}_{r}^{H}(K)$ denotes the set of admissible rooted spanning trees in $\mathcal{T}^{H}(K)$ having $r$ as the root.

A game with hypergraph communication structure, or hypergraph game, is a triple $(N, v, H)$, or shortly $(v, H)$, where $v \in \mathcal{G}_{N}$ is a TU game and $H \in \mathcal{H}_{N}$ is a hypergraph on $N$. When $H$ is a graph $\Gamma$ on $N,(v, \Gamma)$ is a graph game. For fixed player set $N, \mathcal{G}_{N}^{\mathcal{H}}$ $\left(\mathcal{G}_{N}^{\Gamma}\right)$ denotes the set of hypergraph (graph) games, $\mathcal{G}_{N}^{\mathcal{H}^{c}}\left(\mathcal{G}_{N}^{\Gamma^{c}}\right)$ the set of connected hypergraph (graph) games, and $\mathcal{G}_{N}^{\mathcal{H}^{c f}}\left(\mathcal{G}_{N}^{\Gamma^{c f}}\right)$ the set of cycle-free hypergraph (graph) games. The hypergraph-restricted game of a hypergraph game $(v, H) \in \mathcal{G}_{N}^{\mathcal{H}}$ is the TU game $v^{H} \in \mathcal{G}_{N}$, where $v^{H}(S)=\sum_{Q \in S / H} v(Q)$ for all $S \in 2^{N}$. A payoff vector is a vector $x \in \mathbb{R}^{n}$ that assigns payoff $x_{i}$ to player $i \in N$. For a subset of hypergraph games $\mathcal{G} \subseteq \mathcal{G}_{N}^{\mathcal{H}}$, a value on $\mathcal{G}$ is a mapping $\xi: \mathcal{G} \rightarrow \mathbb{R}^{n}$ that assigns to every $(v, H) \in \mathcal{G}$ a payoff vector $\xi(v, H) \in \mathbb{R}^{n}$ with $\xi_{i}(v, H)$ as the payoff to player $i \in N$.

Following Myerson (1980) it is assumed that in a game with hypergraph communication structure each player can communicate with himself and all other players in a hyperlink he belongs to, moreover, all players of a hyperlink have to be present 
before communication between its members can take place. Therefore, only coalitions that are connected in the hypergraph are able to communicate in order to cooperate and realize their worth. A connected coalition in a hypergraph is either a singleton player or a single hyperlink or the connected union of two or more hyperlinks in the hypergraph. The set of connected coalitions in a hypergraph is a building set ${ }^{2}$, cf. Koshevoy and Talman (2014). Note that different hypergraphs may have the same set of connected coalitions.

For a hypergraph game $(v, H) \in \mathcal{G}_{N}^{\mathcal{H}}$ and component $K \in N / H$, the marginal contribution of player $i \in K$ corresponding to admissible rooted spanning tree $T \in$ $\mathcal{T}^{H}(K)$ is given by

$$
m_{i}^{T}(v, H)=v\left(\bar{S}_{i}^{T}\right)-\sum_{Q \in S_{i}^{T} / H} v(Q) .
$$

Since $S_{i}^{T} / H=\left\{\bar{S}_{j}^{T}\right\}_{j \in \widehat{S}_{i}^{T}}$, for every $i \in K$ it holds that

$$
m_{i}^{T}(v, H)=v\left(\bar{S}_{i}^{T}\right)-\sum_{j \in \widehat{S}_{i}^{T}} v\left(\bar{S}_{j}^{T}\right),
$$

being player $i$ 's contribution in worth to his immediate successors and their successors in the tree.

The average tree value (AT value) for hypergraph games, introduced for graph games in Herings et al. $(2008,2010)$ and generalized for hypergraph games in Kang et al. (2020), assigns to every $(v, H) \in \mathcal{G}_{N}^{H}$ a payoff vector $A T(v, H)$ given by

$$
A T_{i}(v, H)=\frac{1}{\left|\mathcal{T}^{H}(K)\right|} \sum_{T \in \mathcal{T}^{H}(K)} m_{i}^{T}(v, H), \quad i \in K, K \in N / H,
$$

being player $i$ 's average marginal contribution corresponding to all admissible rooted spanning trees on the component the player belongs to. In particular, to a graph game $(v, \Gamma) \in \mathcal{G}_{N}^{\Gamma}$, the AT-value assigns the payoff vector $A T(v, \Gamma)$ given by

$$
A T_{i}(v, \Gamma)=\frac{1}{\left|\mathcal{T}^{\Gamma}(K)\right|} \sum_{T \in \mathcal{T}^{\Gamma}(K)} m_{i}^{T}(v, \Gamma), \quad i \in K, K \in N / \Gamma .
$$

- A value $\xi$ satisfies component efficiency $(C E)$ on $\mathcal{G} \subseteq \mathcal{G}_{N}^{\mathcal{H}}$, if for every $(v, H) \in \mathcal{G}$ and $K \in N / H$ it holds that $\sum_{i \in K} \xi_{i}(v, H)=v(K)$.

- A value $\xi$ satisfies component fairness $(C F)$ on $\mathcal{G} \subseteq \mathcal{G}_{N}^{\mathcal{H}}$, if for every $(v, H) \in \mathcal{G}$ and $e \in H$ it holds that

$$
\frac{1}{|K|} \sum_{h \in K}\left(\xi_{h}(v, H)-\xi_{h}(v, H \backslash\{e\})\right)=\frac{1}{\left|K^{\prime}\right|} \sum_{h \in K^{\prime}}\left(\xi_{h}(v, H)-\xi_{h}(v, H \backslash\{e\})\right),
$$

for all distinct $K, K^{\prime} \in N /(H \backslash\{e\})$ satisfying $K \cap e \neq \emptyset$ and $K^{\prime} \cap e \neq \emptyset$.

The average tree value is component efficient and on the subclass of cycle-free (hyper)graph games it is the unique solution that satisfies both component efficiency and component fairness, see Herings et al. (2008) and Kang et al. (2020).

\footnotetext{
${ }^{2}$ A collection of coalitions $\mathcal{B}$ on $N$ is a building set on $N$ if $(i)$ for any $S, Q \in \mathcal{B}$ such that $S \cap Q \neq \emptyset$ it holds that $S \cup Q \in \mathcal{B}$, and $(i i)\{i\} \in \mathcal{B}$ for all $i \in \mathcal{B}$, and therefore, it is also a union stable system, cf. Algaba et al. (2001).
} 


\section{The two-step average tree value}

\subsection{Motivation and definition}

We introduce a two-step average tree value for hypergraph games, and in particular for graph games, which similar to the average tree value for graph and hypergraph games is based on the idea that the payoff to a player is determined by averaging of the player's marginal contributions with respect to all admissible rooted spanning trees of the given communication structure. Since in general for distinct players the numbers of admissible rooted spanning trees having these players as the roots might be different, the simultaneous averaging over all admissible rooted spanning trees unwittingly implies that the players, being the roots of admissible rooted spanning trees, participate in the definition of the average tree value with weights determined by the numbers of admissible rooted spanning trees having the corresponding players as their roots. The latter in a sense conflicts with the idea that every player in a (hyper)graph game is equally important. To eliminate this drawback we consider a two-step averaging procedure, in which in the first step for each player the average of players' marginal contributions corresponding to all admissible rooted spanning trees having this player as the root is calculated, and in the second step the average over all players of all the payoffs obtained in the first step is computed. As we can see later from the comparative analysis of both average tree solution concepts, the better structured averaging procedure underlying the two-step average tree value provides it with additional attractive properties valid on wider subclasses of hypergraph games.

The two-step average tree value (TAT value) for hypergraph games assigns to every $(v, H) \in \mathcal{G}_{N}^{\mathcal{H}}$ a payoff vector $T A T(v, H)$ given by

$$
\operatorname{TAT}_{i}(v, H)=\frac{1}{|K|} \sum_{r \in K} \frac{1}{\left|\mathcal{T}_{r}^{H}(K)\right|} \sum_{T \in \mathcal{T}_{r}^{H}(K)} m_{i}^{T}(v, H), \quad i \in K, K \in N / H .
$$

In particular, to a graph game $(v, \Gamma) \in \mathcal{G}_{N}^{\Gamma}$, the TAT value assigns the payoff vector $T A T(v, \Gamma)$ given by

$$
\operatorname{TAT}_{i}(v, \Gamma)=\frac{1}{|K|} \sum_{r \in K} \frac{1}{\left|\mathcal{T}_{r}^{\Gamma}(K)\right|} \sum_{T \in \mathcal{T}_{r}^{\Gamma}(K)} m_{i}^{T}(v, \Gamma), \quad i \in K, K \in N / \Gamma .
$$

\subsection{The TAT value versus the AT value}

The following example shows that the TAT value may differ from the AT value.

Example 1 Consider the graph game $(v, \Gamma) \in \mathcal{G}_{N}^{\Gamma}$ on a set $N$ of 4 players with $v=u_{\{1,2\}}$ and $\Gamma=\left\{\ell_{1}, \ell_{2}, \ell_{3}, \ell_{4}, \ell_{5}\right\}$, where $\ell_{1}=\{1,2\}, \ell_{2}=\{1,3\}, \ell_{3}=\{1,4\}$, $\ell_{4}=\{2,3\}, \ell_{5}=\{3,4\}$, as depicted in Figure 1 .

As depicted in Figure 2, $\Gamma$ has three admissible rooted spanning trees with player 1 as the root, four with player 2 as the root, three with player 3 as the root, and four with player 4 as the root. 


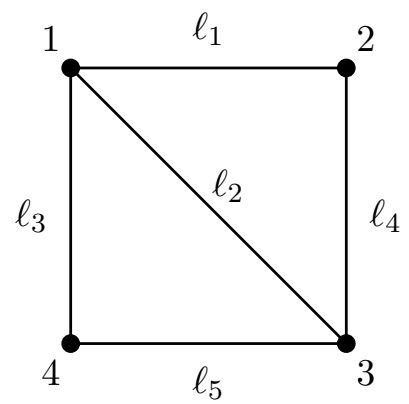

Figure 1: The graph $\Gamma$ in Example 1

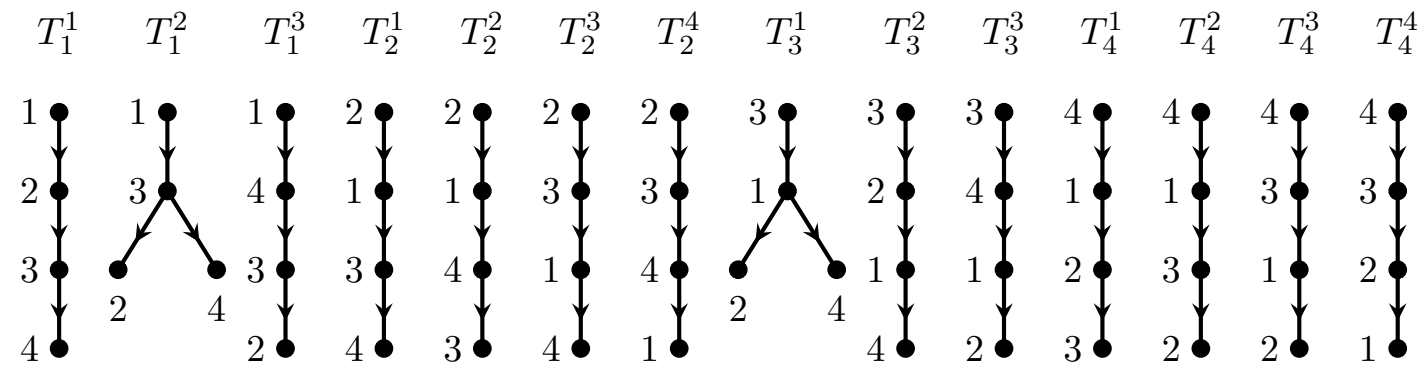

Figure 2: The admissible rooted spanning trees of $\Gamma$ in Example 1

From (1) we obtain the following fourteen marginal contribution vectors:

$$
\begin{aligned}
& m^{T_{1}^{1}}(v, \Gamma)=m^{T_{1}^{2}}(v, \Gamma)=m^{T_{1}^{3}}(v, \Gamma)=(1,0,0,0), \\
& m^{T_{2}^{1}}(v, \Gamma)=m^{T_{2}^{2}}(v, \Gamma)=m^{T_{2}^{3}}(v, \Gamma)=m^{T_{2}^{4}}(v, \Gamma)=(0,1,0,0), \\
& m^{T_{3}^{1}}(v, \Gamma)=m^{T_{3}^{3}}(v, \Gamma)=(1,0,0,0), m^{T_{3}^{2}}(v, \Gamma)=(0,1,0,0), \\
& m^{T_{4}^{1}}(v, \Gamma)=m^{T_{4}^{2}}(v, \Gamma)=m^{T_{4}^{3}}(v, \Gamma)=(1,0,0,0), m^{T_{4}^{4}}(v, \Gamma)=(0,1,0,0) .
\end{aligned}
$$

Wherefrom,

$$
T A T(v, \Gamma) \stackrel{(5)}{=} \frac{1}{4}\left((1,0,0,0)+(0,1,0,0)+\left(\frac{2}{3}, \frac{1}{3}, 0,0\right)+\left(\frac{3}{4}, \frac{1}{4}, 0,0\right)\right)=\left(\frac{29}{48}, \frac{19}{48}, 0,0\right)
$$

and

$$
A T(v, \Gamma) \stackrel{(3)}{=} \frac{1}{14}(8(1,0,0,0)+6(0,1,0,0))=\left(\frac{4}{7}, \frac{3}{7}, 0,0\right)
$$

In some specific cases the TAT and AT values coincide.

Theorem 1 The TAT and AT values for a hypergraph game coincide if each component in the underlying hypergraph is cycle-free, a linear cactus with cycles, or a complete graph.

Proof. Without loss of generality assume that the underlying hypergraph $H \in \mathcal{H}_{N}$ is connected. Let $H$ be a linear cactus without or with cycles. Recall that a connected cycle-free hypergraph is a linear cactus without cycles. We first show that $\left|\mathcal{T}_{r}^{H}(N)\right|=$ $2^{c}$ for all $r \in N$, where $c \in \mathbb{N} \cup\{0\}$ is the number of cycles in $H$. Since two different cycles in $H$ have at most one player in common, the number $c$ is well defined. We 
prove the assertion by induction on the number of cycles. If $H$ has no cycles and therefore $c=0$, it is obvious that $\left|\mathcal{T}_{r}^{H}(N)\right|=1$ and therefore $\left|\mathcal{T}_{r}^{H}(N)\right|=2^{0}$ for all $r \in N$.

Assume that this assertion holds true for every linear cactus with less than $c$ cycles for some $c>0$. We show that the assertion holds true for any linear cactus $H \in \mathcal{H}_{N}$ with $c$ cycles as well. Take any $r \in N$ and $T \in \mathcal{T}_{r}^{H}(N)$. Since $T$ is a rooted tree on $N$, there exists a unique $i \in N$ such that $\bar{S}_{i}^{T}$ is minimal among $\bar{S}_{j}^{T}, j \in N$, containing all $c$ cycles in $H$. For every $j \in N \backslash S_{i}^{T}$, in particular for $j=i$, it holds that $\bar{S}_{j}^{T^{\prime}}=\bar{S}_{j}^{T}$ for all $T^{\prime} \in \mathcal{T}_{r}^{H}(N)$. Therefore, $\left|\mathcal{T}_{r}^{H}(N)\right|=\left|\mathcal{T}_{i}^{H}\left(\bar{S}_{i}^{T}\right)\right|$. To prove that $\left|\mathcal{T}_{i}^{H}\left(\bar{S}_{i}^{T}\right)\right|=2^{c}$, let the components in $S_{i}^{T} / H$ be denoted by $K_{1}, \ldots, K_{s}, K_{s+1}, \ldots, K_{t}$, where $K_{j}$, $j \in\{1, \ldots, s\}$, is such that one of the cycles in $\left.H\right|_{K_{j} \cup\{i\}}$ contains $i$, and where $K_{j}$, $j \in\{s+1, \ldots, t\}$, is such that no cycle in $\left.H\right|_{K_{j} \cup\{i\}}$, if any, contains $i$. For $j=1, \ldots, t$ let $c_{j}$ denote the number of cycles in $\left.H\right|_{K_{j}}$, then $\sum_{j=1}^{s}\left(c_{j}+1\right)+\sum_{j=s+1}^{t} c_{j}=c$ and $0 \leq c_{j}<c$ for all $j \in\{1, \ldots, t\}$. Note that $t \geq 2$ when $s=0$, because $\bar{S}_{i}^{T}$ is the minimal successor set in $T$ containing all cycles in $H$.

Let $F_{i}^{T}=\left\{j \in S_{i}^{T}|i, j \in e, e \in H|_{\bar{S}_{i}^{T}}\right\}$ be the set of successors of $i$ in $T$ that are adjacent to $i$ in $\left.H\right|_{\bar{S}_{i}^{T}}$. Note that $F_{i}^{T}=F_{i}^{T^{\prime}}$ for all $T^{\prime} \in \mathcal{T}_{r}^{H}(N)$ and denote this set by $F_{i}^{r}$. Since $H$ is a linear cactus, it holds that $\left|K_{j} \cap F_{i}^{r}\right|=2$ for $j=1, \ldots, s$ and $\left|K_{j} \cap F_{i}^{r}\right|=1$ for $j=s+1, \ldots, t$. For $j \in\{1, \ldots, s\}$ and with $K_{j} \cap F_{i}^{r}=\{h, k\}$, it follows from the induction argument and the fact that $\left.H\right|_{K_{j}}$ is a linear cactus with $c_{j}<c$ cycles that $\left|\mathcal{T}_{h}^{H}\left(K_{j}\right)\right|=2^{c_{j}}$ and $\left|\mathcal{T}_{k}^{H}\left(K_{j}\right)\right|=2^{c_{j}}$, and therefore $\left|\mathcal{T}_{i}^{H}\left(K_{j} \cup\{i\}\right)\right|=2^{c_{j}+1}$. For $j \in\{s+1, \ldots, t\}$ and with $K_{j} \cap F_{i}^{r}=\{h\}$, it follows from the induction argument and the fact that $\left.H\right|_{K_{j}}$ is a linear cactus with $c_{j}<c$ cycles that $\left|\mathcal{T}_{h}^{H}\left(K_{j}\right)\right|=2^{c_{j}}$, and therefore $\left|\mathcal{T}_{i}^{H}\left(K_{j} \cup\{i\}\right)\right|=2^{c_{j}}$. Since the rooted trees in $\mathcal{T}_{i}^{H}\left(K_{j} \cup\{i\}\right), j=1, \ldots, t$, can be chosen in any combination to be the rooted subtrees with root $i$ of admissible rooted spanning trees in $\mathcal{T}_{i}^{H}\left(\bar{S}_{i}^{T}\right)$, it holds that

$$
\left|\mathcal{T}_{i}^{H}\left(\bar{S}_{i}^{T}\right)\right|=\prod_{j=1}^{t}\left|\mathcal{T}_{i}^{H}\left(K_{j} \cup\{i\}\right)\right|=2^{c} .
$$

This shows that $\left|\mathcal{T}_{r}^{H}(N)\right|=2^{c}$ for all $r \in N$ and therefore $\left|\mathcal{T}^{H}(N)\right|=\sum_{r \in N}\left|\mathcal{T}_{r}^{H}(N)\right|=$ $2^{c} n$. Hence, for a hypergraph game $(v, H) \in \mathcal{G}_{N}^{H}$ with $H$ being a linear cactus with $c$ cycles, it holds that

$$
\begin{aligned}
T A T(v, H) & =\frac{1}{n} \sum_{r \in N} \frac{1}{2^{c}} \sum_{T \in \mathcal{T}_{r}^{H}(N)} m^{T}(v, H) \\
& =\frac{1}{2^{c} n} \sum_{T \in \mathcal{T}^{H}(N)} m^{T}(v, H) \\
& =A T(v, H) .
\end{aligned}
$$

Finally, for a graph game $\left(v, \Gamma^{N}\right) \in \mathcal{G}_{N}^{\Gamma}$ with complete graph it holds that $\left|\mathcal{T}_{r}^{\Gamma^{N}}(N)\right|=$ 
$(n-1)$ ! for all $r \in N$ and $\left|\mathcal{T}^{\Gamma^{N}}(N)\right|=n$ ! and therefore

$$
\begin{aligned}
\operatorname{TAT}\left(v, \Gamma^{N}\right) & =\frac{1}{n} \sum_{r \in N} \frac{1}{(n-1) !} \sum_{T \in \mathcal{T}_{r}^{\Gamma^{N}}(N)} m^{T}\left(v, \Gamma^{N}\right) \\
& =\frac{1}{n !} \sum_{T \in \mathcal{T}^{\Gamma^{N}}(N)} m^{T}\left(v, \Gamma^{N}\right) \\
& =A T\left(v, \Gamma^{N}\right) .
\end{aligned}
$$

If the underlying communication structure is the complete graph, the TAT and AT values for a graph game $\left(v, \Gamma^{N}\right) \in \mathcal{G}_{N}^{\Gamma}$ coincide with the Shapley value for the TU game $v$.

\section{Properties of the TAT value}

The TAT value for hypergraph games, similar to the AT value, meets component efficiency on the entire class of hypergraph games $\mathcal{G}_{N}^{\mathcal{H}}$.

Theorem 2 The TAT value on $\mathcal{G}_{N}^{\mathcal{H}}$ satisfies $C E$.

Proof. Take any $(v, H) \in \mathcal{G}_{N}^{H}$ and $K \in N / H$. From (1) it follows that $\sum_{h \in K} m_{h}^{T}(v, H)=$ $v(K)$ for all $T \in \mathcal{T}^{H}(K)$. Hence,

$$
\begin{aligned}
\sum_{h \in K} T A T_{h}(v, H) & =\sum_{h \in K} \frac{1}{K} \sum_{r \in K} \frac{1}{\left|\mathcal{T}_{r}^{H}(K)\right|} \sum_{T \in \mathcal{T}_{r}^{H}(K)} m_{h}^{T}(v, H) \\
& =\frac{1}{K} \sum_{r \in K} \frac{1}{\left|\mathcal{T}_{r}^{H}(K)\right|} \sum_{T \in \mathcal{T}_{r}^{H}(K)} \sum_{h \in K} m_{h}^{T}(v, H) \\
& =\frac{1}{K} \sum_{r \in K} \frac{1}{\left|\mathcal{T}_{r}^{H}(K)\right|} \sum_{T \in \mathcal{T}_{r}^{H}(K)} v(K)=v(K) .
\end{aligned}
$$

Moreover, the TAT value on $\mathcal{G}_{N}^{\mathcal{H}}$ satisfies the total cooperation equal treatment property.

- A value $\xi$ satisfies the total cooperation equal treatment property (TCETP) on $\mathcal{G} \subseteq \mathcal{G}_{N}^{\mathcal{H}}$, if for every $(v, H) \in \mathcal{G}$ and $K \in N / H$, such that $v(S)=0$ for all $S \in C^{H}(K) \backslash\{K\}$, it holds that $\xi_{i}(v, H)=\xi_{j}(v, H)$ for all $i, j \in K$.

TCETP states that if in a component of the (hyper)graph every proper connected coalition is powerless, then all players in the component get the same payoff. The TCETP is a quite natural property of fair division, which reflects the situation when cooperation is feasible only within an entire component if all players together may contribute in worth of the component they belong to, i.e., the presence of each player is equally important in order to communicate and cooperate, and therefore, all players of the component are rewarded by equal payoffs. 
Theorem 3 The TAT value on $\mathcal{G}_{N}^{H}$ satisfies TCETP.

Proof. Take any $(v, H) \in \mathcal{G}_{N}^{H}$ and $K \in N / H$ satisfying $v(S)=0$ for all $S \in$ $C^{H}(K) \backslash\{K\}$. By (1), for every $T \in \mathcal{T}^{H}(N)$ it holds that

$$
m_{i}^{T}(v, H)= \begin{cases}v(K), & i=r(T) \\ 0, & i \in K \backslash\{r(T)\}\end{cases}
$$

From (4), we obtain

$$
\operatorname{TAT}_{i}(v, H)=\frac{v(K)}{|K|}, i \in K
$$

Example 2 shows that the AT value, different from the TAT value, does not meet TCETP.

Example 2 Consider a graph game $(v, \Gamma) \in \mathcal{G}_{N}^{\Gamma}$ on a set $N$ of 4 players with $v=u_{N}$ and $\Gamma$ as in Example 1. For every $T \in \mathcal{T}^{\Gamma}(N)$ it holds that

$$
m_{i}^{T}(v, \Gamma)= \begin{cases}1, & i=r(T) \\ 0, & i \neq r(T)\end{cases}
$$

Wherefrom, $T A T(v, \Gamma) \stackrel{(5)}{=}\left(\frac{1}{4}, \frac{1}{4}, \frac{1}{4}, \frac{1}{4}\right)$ and $A T(v, \Gamma) \stackrel{(3)}{=}\left(\frac{3}{14}, \frac{2}{7}, \frac{3}{14}, \frac{2}{7}\right)$.

It turns out that the TAT value satisfies component fairness not only on the class of cycle-free hypergraph games, as the AT value, but also on a wider class of hypergraph games, for which the underlying hypergraph is quasi-cycle-free.

A hypergraph $H \in \mathcal{H}_{N}$ is quasi-cycle-free if there exists a cycle-free hypergraph $H^{\prime} \in \mathcal{H}_{N^{\prime}}$ for some $N^{\prime}$ satisfying

(i) $N^{\prime} \subseteq N$ and $\left|H^{\prime}\right|=|H|$;

(ii) $e^{\prime} \in H^{\prime}$ if and only if $e^{\prime}=e \cap N^{\prime}$ for some $e \in H$;

(iii) For every $j \in N \backslash N^{\prime}$ it holds that $\left|H_{j}\right| \geq 2$ and $j \in e_{1} \cap e_{2}$ for some $e_{1}, e_{2} \in H$, $e_{1} \neq e_{2}$, implies $e_{1}^{\prime} \cap e_{2}^{\prime} \neq \emptyset$, where $e_{1}^{\prime}=e_{1} \cap N^{\prime}$ and $e_{2}^{\prime}=e_{2} \cap N^{\prime}$.

From the definition it follows immediately that a cycle-free hypergraph is quasicycle-free. However, a quasi-cycle-free hypergraph may contain cycles. A quasi-cyclefree hypergraph is derived from a cycle-free hypergraph by adding players, if any, to the intersection of hyperlinks. The added players do not change the hyperlink structure of the original cycle-free hypergraph.

Figure 3 depicts in b) a quasi-cycle-free hypergraph $H$ that is not cycle-free and is induced by the cycle-free hypergraph $H^{\prime}$ depicted in a). In $e_{1} \cap e_{2}$ one player from $N \backslash N^{\prime}$ is added to the single player from $e_{1}^{\prime} \cap e_{2}^{\prime}$, and in $e_{1} \cap e_{3}$ two players from $N \backslash N^{\prime}$ are added to the same single player, which belongs also to $e_{1}^{\prime} \cap e_{3}^{\prime}$. Since $\left|e_{1} \cap e_{2}\right|=2$ and $\left|e_{1} \cap e_{3}\right|=3, H$ is not linear, and therefore, has cycles.

For quasi-cycle-free hypergraphs we have the following property. 


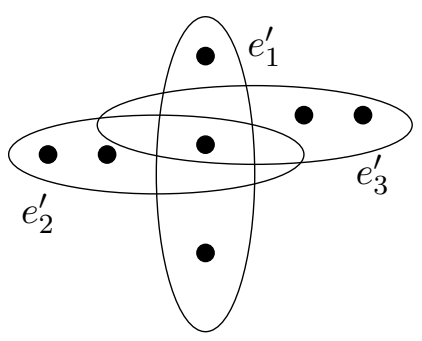

a) $H^{\prime}$

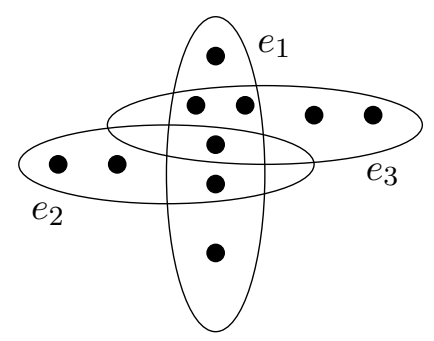

b) $H$

Figure 3: Cycle-free hypergraph $H^{\prime}$ and quasi-cycle-free hypergraph $H$

Lemma 1 Every hyperlink of a quasi-cycle-free hypergraph is a bridge.

Proof. Suppose there exist a quasi-cycle-free hypergraph $H \in \mathcal{H}_{N}$ and a hyperlink $e \in H$ such that $|N / H|=|N /(H \backslash\{e\})|$. Let $K \in N / H$ be such that $\left.e \in H\right|_{K}$. Then $|N / H|=|N /(H \backslash\{e\})|$ implies that $K \in N /(H \backslash\{e\})$, and therefore, $K$ is connected in $H \backslash\{e\}$. Let $H^{\prime}$ on $N^{\prime}$ be a cycle-free hypergraph inducing $H$. Let $e^{\prime}=e \cap N^{\prime}$. Since $e^{\prime} \in H^{\prime},\left|e^{\prime}\right| \geq 2$. Take any $i, j \in e^{\prime}$. Since $K$ is connected in $H \backslash\{e\}$, there exists a chain $\left(i_{1}, e_{1}, i_{2}, \ldots, i_{k-1}, e_{k-1}, i_{k}\right)$ in $H \backslash\{e\}$ between $i$ and $j$ satisfying $i_{1}=i, i_{k}=j$, and $e_{t} \neq e$ for all $t \in\{1, \ldots, k-1\}$. Let $i_{1}^{\prime}=i, i_{k}^{\prime}=j$, $e_{t}^{\prime}=e_{t} \cap N^{\prime}$ for all $t \in\{1, \ldots, k-1\}$, and $i_{t}^{\prime} \in e_{t}^{\prime} \cap e_{t-1}^{\prime}$ for all $t \in\{2, \ldots, k-1\}$. Because $H^{\prime}$ is the inducing cycle-free hypergraph for $H$ and $e_{1}, \ldots, e_{k-1}$ are distinct hyperlinks in $H \backslash\{e\}, e_{1}^{\prime}, \ldots, e_{k-1}^{\prime}$ are distinct hyperlinks in $H^{\prime} \backslash\left\{e^{\prime}\right\}$ and $e_{t}^{\prime} \neq e^{\prime}$ for all $t \in\{1, \ldots, k-1\}$. If all $i_{1}^{\prime}, \ldots, i_{k}^{\prime}$ are distinct, the sequence $\left(i_{1}^{\prime}, e_{1}^{\prime}, i_{2}^{\prime} \ldots, i_{k-1}^{\prime}, e_{k-1}^{\prime}, i_{k}^{\prime}\right)$ is a chain in $H^{\prime} \backslash\left\{e^{\prime}\right\}$ between $i$ and $j$, and $\left(i_{1}^{\prime}, e_{1}^{\prime}, i_{2}^{\prime} \ldots, i_{k-1}^{\prime}, e_{k-1}^{\prime}, i_{k}^{\prime}, e^{\prime}, i_{1}^{\prime}\right)$ is a cycle in $H^{\prime}$, which contradicts that $H^{\prime}$ is cycle-free. Otherwise, if some $i_{1}^{\prime}, \ldots, i_{k}^{\prime}$ coincide, to obtain a chain in $H^{\prime} \backslash\left\{e^{\prime}\right\}$ between $i$ and $j$ we replace in the sequence any subsequence between two identical players by this player. Then by adding $e^{\prime}$ to this chain we obtain again a cycle in $H^{\prime}$, contradicting the cycle-freeness of $H^{\prime}$.

The next theorem states that when the underlying hypergraph is quasi-cycle-free, the TAT value meets CF. From now on the class of quasi-cycle-free hypergraph games on player set $N$ we denote by $\mathcal{G}_{N}^{\mathcal{H}^{q c f}}$.

Theorem 4 The TAT value on $\mathcal{G}_{N}^{\mathcal{H}^{q c f}}$ satisfies $C F$.

Proof. Take any $(v, H) \in \mathcal{G}_{N}^{\mathcal{H}^{q c f}}$ and $e \in H$. Let $K \in N / H$ be such that $\left.e \in H\right|_{K}$. From Lemma 1 it follows that $e$ is a bridge in $H$. Therefore, $K$ consists of at least two components in $H \backslash\{e\}$, denoted by $K^{1}, \ldots, K^{m}$ for some $m \geq 2$. From (1) it follows that for every $T \in \mathcal{T}_{r}^{H}(K), r \in K$, and $j \in\{1, \ldots, m\}$, it holds that

$$
\sum_{i \in K^{j}} m_{i}^{T}(v, H)=v(K)-\sum_{h \neq j} v\left(K^{h}\right), \text { if } r \in K^{j}
$$

and

$$
\sum_{h \in K^{j}} m_{h}^{T}(v, H)=v\left(K^{j}\right), \text { if } r \in K \backslash K^{j}
$$


Therefore, for every $j \in\{1, \ldots, m\}$,

$$
\sum_{r \in K^{j}} \frac{1}{\left|\mathcal{T}_{r}^{H}(K)\right|} \sum_{T \in \mathcal{T}_{r}^{H}(K)} \sum_{i \in K^{j}} m_{i}^{T}(v, H)=\left|K^{j}\right|\left(v(K)-\sum_{h \neq j} v\left(K^{h}\right)\right)
$$

and

$$
\sum_{r \in K \backslash K^{j}} \frac{1}{\left|\mathcal{T}_{r}^{H}(K)\right|} \sum_{T \in \mathcal{T}_{r}^{H}(K)} \sum_{i \in K^{j}} m_{i}^{T}(v, H)=\left|K \backslash K^{j}\right| v\left(K^{j}\right) .
$$

From (4) it follows that for every $j \in\{1, \ldots, m\}$,

$$
\sum_{i \in K^{j}} T A T_{i}(v, H)=\frac{\left|K^{j}\right|\left(v(K)-\sum_{h \neq j} v\left(K^{h}\right)\right)+\left|K \backslash K^{j}\right| v\left(K^{j}\right)}{|K|},
$$

and therefore,

$$
\frac{1}{\left|K^{j}\right|}\left(\sum_{i \in K^{j}} T A T_{i}(v, H)-v\left(K^{j}\right)\right)=\frac{v(K)-\sum_{h=1}^{m} v\left(K^{h}\right)}{|K|} .
$$

By CE $v\left(K^{j}\right)=\sum_{i \in K^{j}} T A T_{i}(v, H \backslash\{e\})$, wherefrom we obtain

$$
\frac{1}{\left|K^{j}\right|} \sum_{i \in K^{j}}\left(T A T_{i}(v, H)-T A T_{i}(v, H \backslash\{e\})\right)=\frac{v(K)-\sum_{h=1}^{m} v\left(K^{h}\right)}{|K|},
$$

which is independent of $j \in\{1, \ldots, m\}$.

However, different from the TAT value, the AT value does not meet $\mathrm{CF}$ on the class $\mathcal{G}_{N}^{\mathcal{H}^{q c f}}$, as illustrated by Example 3 .

Example 3 Consider $(v, H) \in \mathcal{G}_{N}^{\mathcal{H}^{q c f}}$ on a set $N$ of six players with $H=\left\{e_{1}, e_{2}, e_{3}\right\}$, where $e_{1}=\{1,2\}, e_{2}=\{2,3,4,5\}, e_{3}=\{2,3,4,6\}$, as depicted in Figure 4 .

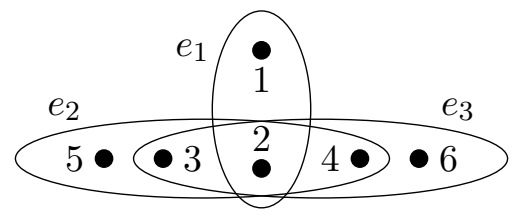

Figure 4: The quasi-cycle-free hypergraph $H$ in Example 3

$H$ contains 10 rooted admissible rooted spanning trees and quite simple calculations provide

$$
A T_{1}(v, H)=\frac{1}{10}(9 v(\{1\})+v(N)-v(N \backslash\{1\})) .
$$

Deleting $e_{1}$ from $H$ splits $N$ into two components, $N /\left(H \backslash\left\{e_{1}\right\}\right)=\left\{K, K^{\prime}\right\}$, where $K=\{1\}$ and $K^{\prime}=N \backslash\{1\}$, and $A T_{1}\left(v, H \backslash\left\{e_{1}\right\}\right)=v(\{1\})$. If the AT value satisfies $\mathrm{CF}$ on $\mathcal{G}_{N}^{\mathcal{H}^{q c f}}$, then $\mathrm{CF}$ requires that

$$
\begin{aligned}
A T_{1}(v, H)-v(\{1\}) & =\frac{1}{5} \sum_{h \in K^{\prime}}\left(A T_{h}(v, H)-A T_{h}\left(v, H \backslash\left\{e_{1}\right\}\right)\right) \\
& \stackrel{(C E)}{=} \frac{1}{5}\left(v(N)-A T_{1}(v, H)-v(N \backslash\{1\})\right),
\end{aligned}
$$


because CE of the AT value implies that $\sum_{i \in N \backslash\{1\}} A T_{i}(v, H)=v(N)-A T_{1}(v, H)$ and $\sum_{i \in N \backslash\{1\}} A T_{i}\left(v, H \backslash\left\{e_{1}\right\}\right)=v(N \backslash\{1\})$. Wherefrom it follows that

$$
A T_{1}(v, H)=\frac{1}{6}(5 v(\{1\})+v(N)-v(N \backslash\{1\})),
$$

which contradicts to the value of $A T_{1}(v, H)$ obtained above.

Furthermore, both the AT and TAT values on the entire class of hypergraph games $\mathcal{G}_{N}^{\mathcal{H}}$ satisfy a rather natural and attractive property of equal surplus of interactive players.

- A value $\xi$ satisfies equal surplus of interactive players $(E S I P)$ on $\mathcal{G} \subseteq \mathcal{G}_{N}^{H}$ if for every $(v, H) \in \mathcal{G}$ and interactive players $i, j$ in $H$ it holds that

$$
\xi_{i}(v, H)-v(\{i\})=\xi_{j}(v, H)-v(\{j\}) .
$$

ESIP states that the players that belong to the same set of hyperlinks, i.e., the players, which can either cooperate with other players only within the same coalitions, or they may stay alone within their own singleton coalitions, receive the same amount of payoff in addition to their own worth. This is a quite reasonable property of fair division. In the next section ESIP will be used also together with $\mathrm{CE}$ and $\mathrm{CF}$ to characterize the TAT value on a particular subclass of semi-cycle-free hypergraph games, the underlying hypergraphs for which may contain cycles.

Before stating the next lemma and theorem, we introduce some extra notation. For a hypergraph $H \in \mathcal{H}_{N}$, component $K \in N / H, r \in K$, and distinct $i, j \in K$, let $\mathcal{T}_{r,(i, j)}^{H}(K)=\left\{T \in \mathcal{T}_{r}^{H}(K) \mid j \in S_{i}^{T}\right\}$ and $\mathcal{T}_{r,\{i, j\}}^{H}(K)=\left\{T \in \mathcal{T}_{r}^{H}(K) \mid \bar{S}_{j}^{T} \cap \bar{S}_{i}^{T}=\right.$ $\emptyset\}$. Note that $\mathcal{T}_{i,(j, i)}^{H}(K)=\mathcal{T}_{i,\{i, j\}}^{H}(K)=\emptyset$ and $\mathcal{T}_{i,(i, j)}^{H}(K)=\mathcal{T}_{i}^{H}(K), \mathcal{T}_{j,(i, j)}^{H}(K)=$ $\mathcal{T}_{j,\{i, j\}}^{H}(K)=\emptyset$ and $\mathcal{T}_{j,(j, i)}^{H}(K)=\mathcal{T}_{j}^{H}(K)$, and $\left\{\mathcal{T}_{r,(i, j)}^{H}(K), \mathcal{T}_{r,(j, i)}^{H}(K), \mathcal{T}_{r,\{i, j\}}^{H}(K)\right\}$ is a partition of $\mathcal{T}_{r}^{H}(K)$ for all $r \in K \backslash\{i, j\}$.

Lemma 2 For every $H \in \mathcal{H}_{N}$ and interactive players $i, j \in K, K \in N / H$, it holds that $\left|\mathcal{T}_{i}^{H}(K)\right|=\left|\mathcal{T}_{j}^{H}(K)\right|$ and $\left|\mathcal{T}_{r,(i, j)}^{H}(K)\right|=\left|\mathcal{T}_{r,(j, i)}^{H}(K)\right|$ for all $r \in K \backslash\{i, j\}$.

Proof. Take any $r \in K \backslash\{j\}$ and $T \in \mathcal{T}_{r,(i, j)}^{H}(K)$. Recall that $\mathcal{T}_{i,(i, j)}^{H}(K)=\mathcal{T}_{i}^{H}(K)$. Since $T$ is an admissible rooted spanning tree of $\left.H\right|_{K}, H_{i}=H_{j}$, and $j \in S_{i}^{T}$, we have that $(i, j) \in T$ and $\bar{S}_{j}^{T}=\{j\}$. Let the rooted tree $T^{\prime}$ on $K$ be given by

$$
\bar{S}_{h}^{T^{\prime}}= \begin{cases}\bar{S}_{i}^{T}, & \text { if } h=j, \\ \{i\}, & \text { if } h=i, \\ \overline{S_{h}^{T},} & \text { if } h \in K \backslash\{i, j\} .\end{cases}
$$

Since $H_{i}=H_{j}$, we have that $(j, i) \in T^{\prime}$ and therefore $T^{\prime} \in \mathcal{T}_{j}^{H}(K)$ if $r=i$ and $T^{\prime} \in \mathcal{T}_{r,(j, i)}^{H}(K)$ if $r \in K \backslash\{i, j\}$.

Reversely, take any $r \in K \backslash\{i\}$ and $T^{\prime} \in \mathcal{T}_{r,(j, i)}^{H}(K)$. Recall that $\mathcal{T}_{j,(j, i)}^{H}(K)=$ $\mathcal{T}_{j}^{H}(K)$. Then there exists a unique $T \in \mathcal{T}_{i}^{H}(K)$ if $r=j$ and $T \in \mathcal{T}_{r,(i, j)}^{H}(K)$ if $r \in$ 
$K \backslash\{i, j\}$ satisfying (6). Therefore, $\left|\mathcal{T}_{i}^{H}(K)\right|=\left|\mathcal{T}_{j}^{H}(K)\right|$ and $\left|\mathcal{T}_{r,(i, j)}^{H}(K)\right|=\left|\mathcal{T}_{r,(j, i)}^{H}(K)\right|$ for all $r \in K \backslash\{i, j\}$.

The lemma shows that for any two given interactive players $i$ and $j$ in some component $K$ in a hypergraph $H$, there are always pairwise two admissible rooted spanning trees $T$ and $T^{\prime}$ in $\mathcal{T}^{H}(K)$ for which only the sets of successors of $i$ and $j$ differ from each other. Therefore, the number of admissible rooted spanning trees for which $i$ has $j$ as successor equals the number of admissible rooted spanning trees for which $j$ has $i$ as successor. Moreover, since $\bar{S}_{h}^{T}=\bar{S}_{h}^{T^{\prime}}$ for all $h \in K \backslash\{i, j\}$, it holds that $\left(S_{i}^{T} \backslash\{j\}\right) / H=\left(S_{j}^{T^{\prime}} \backslash\{i\}\right) / H$.

Next we show that on the entire class of hypergraph games both the AT and TAT values satisfy ESIP.

Theorem 5 The AT and TAT values on $\mathcal{G}_{N}^{\mathcal{H}}$ satisfy ESIP.

Proof. Take any $(v, H) \in \mathcal{G}_{N}^{\mathcal{H}}$ and interactive players $i, j \in N$ in $H$. Let $K \in N / H$ be such that $i, j \in K$ and let $B=\left\{h \in K \mid H_{h}=H_{i}\right\}$ be the set of interactive players in $H$ that contains $i$ and $j$. We will prove that

$$
A T_{i}(v, H)-A T_{j}(v, H)=v(\{i\})-v(\{j\})
$$

and

$$
T A T_{i}(v, H)-T A T_{j}(v, H)=v(\{i\})-v(\{j\}) .
$$

Take any $T \in \mathcal{T}_{i}^{H}(K)$. Since $\mathcal{T}_{i,(i, j)}^{H}(K)=\mathcal{T}_{i}^{H}(K)$, it holds that $T \in \mathcal{T}_{i,(i, j)}^{H}(K)$. Let $T^{\prime} \in \mathcal{T}_{j,(j, i)}^{H}(K)$ be defined as in (6). Then, $\bar{S}_{i}^{T}=\bar{S}_{j}^{T^{\prime}}=K,\{h\} \in S_{i}^{T} / H$ for all $h \in B \backslash\{i\}$, and $\{h\} \in S_{j}^{T^{\prime}} / H$ for all $h \in B \backslash\{j\}$. Whence together with (1) it follows

$$
m_{i}^{T}(v, H)=v(K)-\sum_{h \in B \backslash\{i\}} v(\{h\})-\sum_{Q \in(K \backslash B) / H} v(Q)
$$

and

$$
m_{j}^{T^{\prime}}(v, H)=v(K)-\sum_{h \in B \backslash\{j\}} v(\{h\})-\sum_{Q \in(K \backslash B) / H} v(Q),
$$

with difference $v(\{i\})-v(\{j\})$. Moreover, $m_{i}^{T^{\prime}}(v, h)=v(\{i\})$ and $m_{j}^{T}(v, H)=v(\{j\})$, also with difference $v(\{i\})-v(\{j\})$. Since, by Lemma $2,\left|\mathcal{T}_{i}^{H}(K)\right|=\left|\mathcal{T}_{j}^{H}(K)\right|$, we obtain

$$
\frac{1}{\left|\mathcal{T}_{i}^{H}(K)\right|} \sum_{T \in \mathcal{T}_{i}^{H}(K)} m_{i}^{T}(v, H)-\frac{1}{\left|\mathcal{T}_{j}^{H}(K)\right|} \sum_{T \in \mathcal{T}_{j}^{H}(K)} m_{j}^{T}(v, H)=v(\{i\})-v(\{j\})
$$

and

$$
\frac{1}{\left|\mathcal{T}_{j}^{H}(K)\right|} \sum_{T \in \mathcal{T}_{j}^{H}(K)} m_{i}^{T}(v, H)-\frac{1}{\left|\mathcal{T}_{i}^{H}(K)\right|} \sum_{T \in \mathcal{T}_{i}^{H}(K)} m_{j}^{T}(v, H)=v(\{i\})-v(\{j\}) .
$$

Next, take any $r \in K \backslash\{i, j\}$ and $T \in \mathcal{T}_{r,(i, j)}^{H}(K)$ and let $T^{\prime} \in \mathcal{T}_{r,(j, i)}^{H}(K)$ be as defined in (6). Then, $\bar{S}_{i}^{T}=\bar{S}_{j}^{T^{\prime}},\{h\} \in S_{i}^{T} / H$ for all $h \in B \backslash\{i\}$, and $\{h\} \in S_{j}^{T^{\prime}} / H$ for all $h \in B \backslash\{j\}$. Whence together with (1) it follows

$$
m_{i}^{T}(v, H)=v\left(\bar{S}_{i}^{T}\right)-\sum_{h \in B \backslash\{i\}} v(\{h\})-\sum_{Q \in\left(\bar{S}_{i}^{T} \backslash B\right) / H} v(Q)
$$


and

$$
m_{j}^{T^{\prime}}(v, H)=v\left(\bar{S}_{j}^{T^{\prime}}\right)-\sum_{h \in B \backslash\{j\}} v(\{h\})-\sum_{Q \in\left(\bar{S}_{j}^{\left.T^{\prime} \backslash B\right) / H}\right.} v(Q),
$$

with difference $v(\{i\})-v(\{j\})$. Moreover, $m_{i}^{T^{\prime}}(v, H)=v(\{i\})$ and $m_{j}^{T}(v, H)=v(\{j\})$, also with difference $v(\{i\})-v(\{j\})$. And, if we take $T \in \mathcal{T}_{r,\{i, j\}}^{H}(K)$, then there exists $h \in K \backslash\{i, j\}$ satisfying $\{i\},\{j\} \in S_{h}^{T} / H$ and therefore $m_{i}^{T}(v, H)=v(\{i\})$ and $m_{j}^{T}(v, H)=v(\{j\})$, again with difference $v(\{i\})-v(\{j\})$.

Since for every $r \in K \backslash\{i, j\}$ it holds that $\left\{\mathcal{T}_{r,(i, j)}^{H}(K), \mathcal{T}_{r,(j, i)}^{H}(K), \mathcal{T}_{r,\{i, j\}}^{H}(K)\right\}$ is a partition of $\mathcal{T}_{r}^{H}(K)$ and, by Lemma $2,\left|\mathcal{T}_{r,(i, j)}^{H}(K)\right|=\left|\mathcal{T}_{r,(j, i)}^{H}(K)\right|$, we obtain

$$
\frac{1}{\left|\mathcal{T}_{r}^{H}(K)\right|} \sum_{T \in \mathcal{T}_{r}^{H}(K)} m_{i}^{T}(v, H)-\frac{1}{\left|\mathcal{T}_{r}^{H}(K)\right|} \sum_{T \in \mathcal{T}_{r}^{H}(K)} m_{j}^{T}(v, H)=v(\{i\})-v(\{j\})
$$

for all $r \in K \backslash\{i, j\}$.

Taking the average of the $|K|$ differences derived above, we obtain $T A T_{i}(v, H)-$ $T A T_{j}(v, H)=v(\{i\})-v(\{j\})$. In addition, we also have

$$
\frac{1}{\left|\mathcal{T}^{H}(K)\right|} \sum_{T \in \mathcal{T}^{H}(K)} m_{i}^{T}(v, H)-\frac{1}{\left|\mathcal{T}^{H}(K)\right|} \sum_{T \in \mathcal{T}^{H}(K)} m_{j}^{T}(v, H)=v(\{i\})-v(\{j\}) .
$$

Wherefrom by (2) it follows that $A T_{i}(v, H)-A T_{j}(v, H)=v(\{i\})-v(\{j\})$.

\section{An axiomatization of the TAT value}

Since the TAT and the AT values coincide on the subclass of cycle-free (hyper)graph games, the existing axiomatic characterizations of the AT value for cycle-free (hyper)graph games are also valid for the TAT value.

Moreover, we obtain an axiomatization of the TAT value on a specific subclass of the so-called semi-cycle-free hypergraph games, the underlying hypergraphs for which may contain cycles.

A hypergraph $H \in \mathcal{H}_{N}$ is semi-cycle-free if there exists a cycle-free hypergraph $H^{\prime} \in \mathcal{H}_{N^{\prime}}$ for some $N^{\prime}$ satisfying

(i) $N^{\prime} \subseteq N$ and $\left|H^{\prime}\right|=|H|$;

(ii) $e^{\prime} \in H^{\prime}$ if and only if $e^{\prime}=e \cap N^{\prime}$ for some $e \in H$;

$\left(i i i^{\prime}\right)$ For every $j \in N \backslash N^{\prime}$ it holds that $\left|H_{j}\right| \geq 2$ and $H_{j}=H_{i}$ for some $i \in N^{\prime}$.

Obviously, a cycle-free hypergraph is semi-cycle-free and a semi-cycle-free hypergraph is quasi-cycle-free. A semi-cycle-free hypergraph $H$ is derived from a cycle-free hypergraph $H^{\prime}$ by adding players, if any, which become interactive with a connective player in $N^{\prime}$. The added players do not change the hyperlink structure of the original cycle-free hypergraph.

Figure 5 depicts in b) a semi-cycle-free hypergraph $H$ with cycles induced by the cycle-free hypergraph $H^{\prime}$ depicted in a). In $e_{1} \cap e_{2}$ one player from $N \backslash N^{\prime}$ is added 


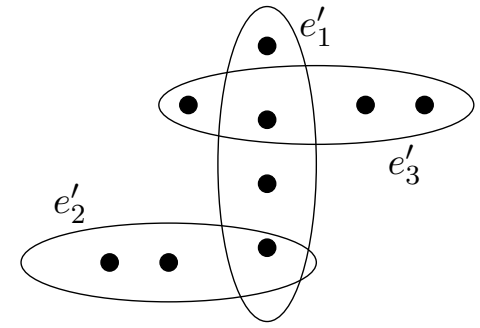

a) $H^{\prime}$

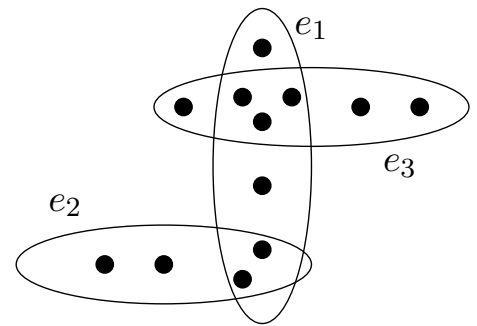

b) $H$

Figure 5: Cycle-free hypergraph $H^{\prime}$ and semi-cycle-free hypergraph $H$

to become interactive with the single player from $N^{\prime}$, and in $e_{1} \cap e_{3}$ two players from $N \backslash N^{\prime}$ are added to become interactive with the single player from $N^{\prime}$.

The additional players in a semi-cycle-free hypergraph $H$ have the same degree as the corresponding players in the inducing cycle-free hypergraph $H^{\prime}$, while this is not necessarily true for the connective players in a quasi-cycle-free hypergraph, as is illustrated by Figure 6 .

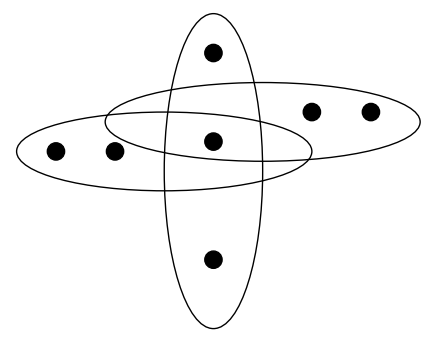

a) Cycle-free

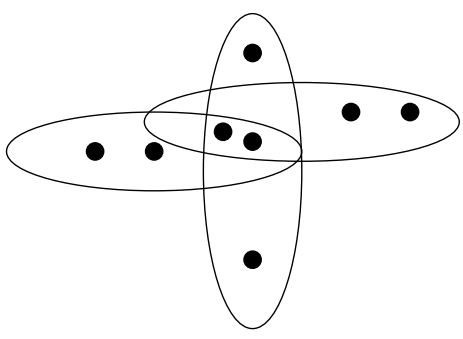

b) Semi-cycle-free

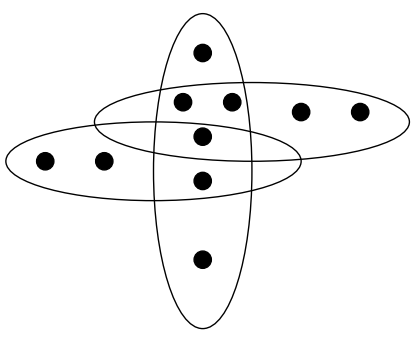

c) Quasi-cycle-free

Figure 6: Three types of hypergraphs

The next theorem shows that the TAT value for semi-cycle-free hypergraph games is characterized by CE, CF, and ESIP. From now on the class of semi-cycle-free hypergraph games on player set $N$ we denote by $\mathcal{G}_{N}^{\mathcal{H}^{\text {scf }}}$.

Theorem 6 The TAT value on $\mathcal{G}_{N}^{\mathcal{H}^{\text {scf }}}$ is uniquely defined by CE, CF, and ESIP.

Proof. From Theorems 2, 4, and 5 it follows that on $\mathcal{G}_{N}^{\mathcal{H}^{s c f}}$ the TAT value satisfies $\mathrm{CE}, \mathrm{CF}$, and ESIP.

To prove the reverse, let $\xi$ be a value on $\mathcal{G}_{N}^{\mathcal{H}^{\text {scf }}}$ that meets $\mathrm{CE}, \mathrm{CF}$, and ESIP. Take any $(v, H) \in \mathcal{G}_{N}^{\mathcal{H}^{s c f}}$ and $K \in N / H$. If $\left.H\right|_{K}=\emptyset$, then $K=\{i\}$ for some $i \in N$ and CE implies $\xi_{i}(v, H)=v(\{i\})$. Assume $\left.H\right|_{K} \neq \emptyset$. Let $H^{\prime} \in \mathcal{H}_{N^{\prime}}$ be a cycle-free hypergraph by which $H$ is induced and let $K^{\prime}=K \cap N^{\prime}$. For every $i \in K^{\prime}$, let $B_{i}=\{i\}$ if $\left|H_{i}\right|=1$ and otherwise $B_{i}=\left\{j \in K \mid H_{j}=H_{i}\right\}$. Let $p_{e}=\left|\left\{B_{i} \subseteq e \mid i \in K^{\prime}\right\}\right|$ for all $\left.e \in H\right|_{K}$. Then $\left|e^{\prime}\right|=p_{e}$, where $e^{\prime}=e \cap N^{\prime}$. Since $H^{\prime}$ is cycle-free, from Lemma 3 in Kang et al. (2020) it follows that

$$
\sum_{\left.e^{\prime} \in H^{\prime}\right|_{K^{\prime}}}\left(\left|e^{\prime}\right|-1\right)=\left|K^{\prime}\right|-1
$$


which implies

$$
\sum_{\left.e \in H\right|_{K}}\left(p_{e}-1\right)=\left|K^{\prime}\right|-1
$$

We show first that there are $\left|K^{\prime}\right|$ linearly independent equations when applying $\mathrm{CE}$ and CF. CE implies that

$$
\sum_{i \in K} \xi_{i}(v, H)=v(K)
$$

and

$$
\sum_{i \in \widehat{K}} \xi_{i}(v, H \backslash\{e\})=v(\widehat{K}), \widehat{K} \in K /(H \backslash\{e\})
$$

for all $\left.e \in H\right|_{K}$. CF implies that, for each $\left.e \in H\right|_{K}$,

$$
\frac{1}{\left|K^{1}\right|}\left(\sum_{h \in K^{1}} \xi_{h}(v, H)-v\left(K^{1}\right)\right)=\frac{1}{\left|K^{2}\right|}\left(\sum_{h \in K^{2}} \xi_{h}(v, H)-v\left(K^{2}\right)\right),
$$

for every $K^{1}, K^{2} \in K /(H \backslash\{e\})$ satisfying $K^{1} \cap e \neq \emptyset$ and $K^{2} \cap e \neq \emptyset$. Therefore, for each $\left.e \in H\right|_{K}$,

$$
\sum_{h \in \widehat{K}} \xi_{h}(v, H)=|\widehat{K}| \alpha_{e}+v(\widehat{K})
$$

for every $\widehat{K} \in K /(H \backslash\{e\})$ satisfying $\widehat{K} \cap e \neq \emptyset$, where

$$
\alpha_{e}=\frac{1}{|K|}\left(v(K)-\sum_{Q \in K /(H \backslash\{e\})} v(Q)\right) .
$$

Note that if $H_{i}=H_{j}=\{e\}$,

$$
\xi_{i}(v, H)-v(\{i\}) \stackrel{(9)}{=} \xi_{j}(v, H)-v(\{j\}),
$$

which agrees also with ESIP. Since $H$ is semi-cycle-free, it holds that $|K /(H \backslash\{e\})|=$ $p_{e}$ for all $\left.e \in H\right|_{K}$.

Equations (7) and (9) yield $\sum_{\left.e \in H\right|_{K}}\left(p_{e}-1\right)+1=\left|K^{\prime}\right|$ linearly independent equations on $|K|$ variables, which implies that the total payoff of $B_{j}, j \in K^{\prime}$, is

$$
\sum_{i \in B_{j}} \xi_{i}(v, H)=a_{j}
$$

where $a_{j}, j \in K^{\prime}$, is some constant.

Next, we examine the payoffs of the players in $B_{h}$ for $h \in K^{\prime}$. When $\left|B_{h}\right|=1$, the payoff of the unique player in $B_{h}$ is determined by equation (10). When $\left|B_{h}\right| \geq 2$, ESIP implies that for every $i, j \in B_{h}$

$$
\xi_{i}(v, H)-v(\{i\})=\xi_{j}(v, H)-v(\{j\}),
$$

yielding $\left|B_{h}\right|-1$ linearly independent equations on $\left|B_{h}\right|$ variables. Combined with equation (10), these equations uniquely determine the payoffs of all players in $B_{h}$, $h \in K^{\prime}$. This implies that $\xi_{i}(v, H)$ is uniquely determined for all $i \in K$ for any $K \in N / H$.

The next example proves logical independence of axioms CE, CF, and ESIP in the axiomatization of the TAT value on $\mathcal{G}_{N}^{\mathcal{H}^{s c f}}$ in Theorem 6 . 
Example 4 (1) Let value $\xi^{1}$ on $\mathcal{G}_{N}^{\mathcal{H}^{s c f}}$ be given by

$$
\xi_{i}^{1}(v, H)=0, \quad \text { for all } i \in N .
$$

The value $\xi^{1}$ satisfies all axioms except $\mathrm{CE}$.

(2) Let value $\xi^{2}$ on $\mathcal{G}_{N}^{\mathcal{H}^{\text {scf }}}$ be given by the Myerson value, cf. van den Nouweland et al. (1992). The value $\xi^{2}$ satisfies all axioms except CF.

(3) Let value $\xi^{3}$ on $\mathcal{G}_{N}^{\mathcal{H}^{\text {scf }}}$ be given by

$$
\xi_{i}^{3}(v, H)= \begin{cases}T A T_{i}(v, H), & \text { if } i \in N \backslash B, \\ \frac{1}{|B|} \sum_{j \in B} T A T_{j}(v, H), & \text { if } i \in B\end{cases}
$$

where $B$ is one of the sets of connective interactive players in $H$, if any exists. The value $\xi^{3}$ satisfies all axioms except ESIP.

\section{Core stability of the TAT value}

Since the TAT and AT values coincide on the subclass of cycle-free (hyper)graph games, then as a corollary to the core stability of the AT value for superadditive cycle-free (hyper)graph games, the TAT value of a cycle-free (hyper)graph game with superadditive underlying TU game is an element of the core. Moreover, it turns out that both the AT and TAT values are core stable also on the wider subclass of quasi-cycle-free superadditive hypergraph games.

The core of a hypergraph game is determined as the set of all component efficient payoff vectors, at which every connected coalition gets at least its own worth. Formally the core of $(v, H) \in \mathcal{G}_{N}^{\mathcal{H}}$ is defined by

$$
C(v, H)=\left\{x \in \mathbb{R}^{n} \mid \sum_{i \in K} x_{i}=v(K), \forall K \in N / H ; \sum_{i \in S} x_{i} \geq v(S), \forall S \in C^{H}(N)\right\} .
$$

Theorem 7 For every $(v, H) \in \mathcal{G}_{N}^{\mathcal{H}^{q c f}}$ with superadditive $v, A T(v, H), T A T(v, H) \in$ $C(v, H)$.

Proof. For $Q \in 2^{N} \backslash\{\emptyset\}$, let $\left.v\right|_{Q}$ denote the subgame of $v$ on $Q$, where $\left.v\right|_{Q}(S)=v(S)$ for all $S \subseteq Q$. Since $S \in C^{H}(N)$ if and only if $S \in C^{\left.H\right|_{K}}(K)$ for some $K \in N / H$, it holds that $x \in C(v, H)$ if and only if $\left(x_{i}\right)_{i \in K} \in C\left(\left.v\right|_{K},\left.H\right|_{K}\right)$ for all $K \in N / H$. We first prove that for every $K \in N / H$ and $T \in \mathcal{T}^{H}(K)$ it holds that $\left(m_{i}^{T}(v, H)\right)_{i \in K} \in$ $C\left(\left.v\right|_{K},\left.H\right|_{K}\right)$.

Take any $K \in N / H$ and $T \in \mathcal{T}^{H}(K)$. From (1) it immediately follows that $\sum_{i \in K} m_{i}^{T}(v, H)=v(K)$. Take any $S \in C^{\left.H\right|_{K}}(K)$. Since $S \subseteq K$ and $T$ is an admissible rooted spanning tree of $\left.H\right|_{K}$, there exist unique $i_{1}, \ldots, i_{k} \in S$ for some $k \geq 1$ such that $S \subseteq \bigcup_{j=1}^{k} \bar{S}_{i_{j}}^{T}, \bar{S}_{i_{j}}^{T} \cap S \neq \emptyset$ for all $j \in\{1, \ldots, k\}$, and $\bar{S}_{i_{1}}^{T}, \ldots, \bar{S}_{i_{k}}^{T}$ are the $k$ distinct components of $\bigcup_{j=1}^{k} \bar{S}_{i_{j}}^{T}$ in $\left.H\right|_{K}$. Because $S$ is connected in $\left.H\right|_{K}$, it must hold that $k=1$. Therefore, there exists a unique $i \in S$ satisfying $S \subseteq \bar{S}_{i}^{T}$. 
Let $\widehat{S}_{S}^{T}=\{j \in K \backslash S \mid(h, j) \in T, h \in S\}$ be the set of immediate successors of $S$ in $T$. Since $H$ is quasi-cycle-free and $S$ is connected in $\left.H\right|_{K}, \bar{S}_{i}^{T}$ is partitioned by the connected coalitions $S$ and $\bar{S}_{j}^{T}, j \in \widehat{S}_{S}^{T}$. Therefore, we have

$$
\begin{aligned}
\sum_{h \in S} m_{h}^{T}(v, H) & =\sum_{h \in S}\left(v\left(\bar{S}_{h}^{T}\right)-\sum_{j \in \widehat{S}_{h}^{T}} v\left(\bar{S}_{j}^{T}\right)\right) \\
& =v\left(\bar{S}_{i}^{T}\right)-\sum_{j \in \widehat{S}_{S}^{T}} v\left(\bar{S}_{j}^{T}\right) \\
& =v\left(S \cup\left(\bigcup_{j \in \widehat{S}_{S}^{T}} \bar{S}_{j}^{T}\right)\right)-\sum_{j \in \widehat{S}_{S}^{T}} v\left(\bar{S}_{j}^{T}\right) \\
& \geq v(S),
\end{aligned}
$$

where the first equality follows from (1), the second equality follows because for every $h \in S \backslash\{i\}$ the first term cancels, the third equality follows from the fact that $\bar{S}_{i}^{T}=S \cup\left(\bigcup_{j \in \widehat{S}_{S}^{T}} \bar{S}_{j}^{T}\right)$, and the inequality follows from repeated application of superadditivity of $v$ and the fact that $\bar{S}_{i}^{T}$ is partitioned by $S$ and $\bar{S}_{j}^{T}, j \in \widehat{S}_{S}^{T}$. Together with $\sum_{h \in K} m_{h}^{T}(v, H)=v(K)$, we obtain $\left(m_{i}^{T}(v, H)\right)_{i \in K} \in C\left(\left.v\right|_{K},\left.H\right|_{K}\right)$.

Since, for every $K \in N / H, C\left(\left.v\right|_{K},\left.H\right|_{K}\right)$ is a convex set and $\left(T A T_{i}(v, H)\right)_{i \in K}$ and $\left(A T_{i}(v, H)\right)_{i \in K}$ are convex combinations of $\left(m_{i}^{T}(v, H)\right)_{i \in K}$ over all $T \in \mathcal{T}^{H}(K)$, we obtain that both $\left(T A T_{i}(v, H)\right)_{i \in K}$ and $\left(A T_{i}(v, H)\right)_{i \in K}$ are elements of $C\left(\left.v\right|_{K},\left.H\right|_{K}\right)$ for all $K \in N / H$.

\section{References}

Algaba, E., Bilbao, J.M., Borm, P., and López, J.J., (2001), The Myerson value for union stable systems, Mathematical Methods of Operations Research, 54(3), 359371.

Borm, P., Owen, G., and Tijs, S., (1992), On the position value for communication situation, SIAM Journal on Discrete Mathematics, 5(3), 305-320.

Herings, P.J.J., van der Laan, G., and Talman, A.J.J. (2008), The average tree solution for cycle-free graph games, Games and Economic Behavior, 62, 77-92.

Herings, P.J.J., van der Laan, G., Talman, A.J.J., and Yang, Z (2010), The average tree solution for cooperative games with communication structure, Games and Economic Behavior, 68, 626-633.

Kang, L., Khmelnitskaya, A.B., Shan, E., Talman, A.J.J., and Zhang G. (2020), The average tree value for hypergraph games, CentER Discussion Paper No.2020-005, Tilburg University, The Netherlands.

Koshevoy, G. and Talman, A.J.J. (2014), Solution concepts for games with general coalitional structure, Mathematical Social Sciences, 68(1), 19-30.

Meessen, R. (1988), Communication Games, Master's thesis, Departartment of Mathematics, University of Nijmegen, the Netherlands (In Dutch). 
Myerson, R.B. (1977), Graphs and cooperation in games, Mathematics of Operations Research, 2, 225-229.

Myerson, R.B. (1980), Conference structures and fair allocation rules, International Journal of Game Theory, 9, 169-182.

van den Nouweland, A., Borm, P., and Tijs, S. (1992), Allocation rules for hypergraph communication situations, International Journal of Game Theory, 20, 255-268.

Shapley, L.S. (1953), A value for $n$-person games, in: Kuhn, H.W., and A.W. Tucker, (eds), Contributions to the Theory of Games II, Princeton: Princeton University Press, pp. 307-317. 